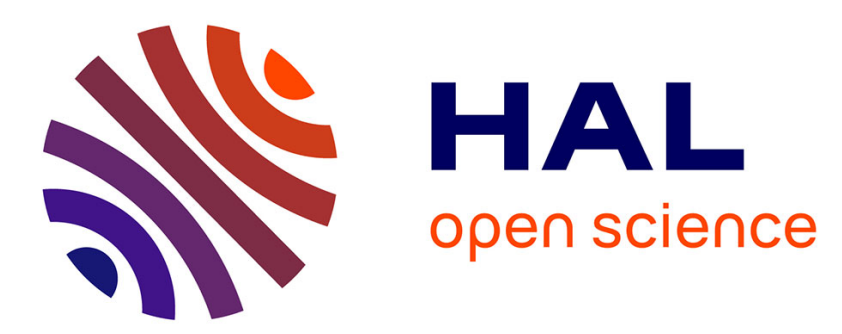

\title{
Growth and characterization of gallium oxide thin films by radiofrequency magnetron sputtering
}

Philippe Marie, Xavier Portier, Julien Cardin

\section{To cite this version:}

Philippe Marie, Xavier Portier, Julien Cardin. Growth and characterization of gallium oxide thin films by radiofrequency magnetron sputtering. physica status solidi (a), 2008, 205 (8), pp.1943-1946. 10.1002/pssa.200778856 . hal-01204711

\section{HAL Id: hal-01204711 \\ https://hal.science/hal-01204711}

Submitted on 3 Apr 2017

HAL is a multi-disciplinary open access archive for the deposit and dissemination of scientific research documents, whether they are published or not. The documents may come from teaching and research institutions in France or abroad, or from public or private research centers.
L'archive ouverte pluridisciplinaire HAL, est destinée au dépôt et à la diffusion de documents scientifiques de niveau recherche, publiés ou non, émanant des établissements d'enseignement et de recherche français ou étrangers, des laboratoires publics ou privés. 


\title{
Growth and characterization of gallium oxide thin films by radiofrequency magnetron sputtering
}

\author{
P. Marie*, X. Portier, and J. Cardin \\ CIMAP-CEA-CNRS-ENSICAEN-Université de Caen, 6 Boulevard Maréchal Juin, 14050 CAEN Cedex 4, France \\ * Corresponding author: e-mail philippe.marie@ensicaen.fr
}

\begin{abstract}
Undoped and Neodymium-doped gallium oxide $\left(\mathrm{Ga}_{2} \mathrm{O}_{3}\right)$ thin films of about $500 \mathrm{~nm}$ thickness were successfully grown at different temperatures ranging from 100 up to $600{ }^{\circ} \mathrm{C}$ by radiofrequency magnetron sputtering. Post-annealing treatments were carried out at $900{ }^{\circ} \mathrm{C}$ and $1000{ }^{\circ} \mathrm{C}$. The obtained films were (400) textured and a grain size of a few tens of
\end{abstract}

nanometres was found. Optical and electrical characterizations led to a figure of merit of about $1.9 \times 10^{-4}$. These films were successfully doped with Neodymium by a co-sputtering method. The photoluminescence experiments for the Nd-doped $\beta-\mathrm{Ga}_{2} \mathrm{O}_{3}$ films clearly showed the rare-earth emitting signature.
1 Introduction Recently, transparent conductive oxide (TCO) as alternative to expensive ITO have focused much attention owing to the wide variety of possible applications such as optical windows [1,2], high temperature chemical gas sensor [3], and dielectric layer [4]. Gallium zinc oxide (GZO) thin films have shown high conductivity and transparent properties with figure of merit comparable to those of ITO materials [5]. Among promising TCO materials, $\mathrm{Ga}_{2} \mathrm{O}_{3}$ is a wide band gap semiconductor material $(4.9 \mathrm{eV})$ and its conductivity can vary from insulator to conductor depending on the growth conditions [1,2]. Over the past few years, by doping this material with rare-earth elements or $3 \mathrm{~d}$ transition metal ions, $\mathrm{Ga}_{2} \mathrm{O}_{3}$ thin films have also shown promising optical and photoluminescent (PL) properties [6] as well as possible applications in electroluminescent devices (thin film electroluminescent displays) [7]. The most suitable structure for applications is the monoclinic $\beta-\mathrm{Ga}_{2} \mathrm{O}_{3}$ phase $(a=1.221 \mathrm{~nm}, b=0.304 \mathrm{~nm}$, $c=0.580 \mathrm{~nm}$ and $\beta=103.8^{\circ}$, space group: $\mathrm{C} 2 / \mathrm{m}$ ) which appears to be extremely stable chemically and thermally (melting point at $1800^{\circ} \mathrm{C}$ ) and may be an alternative choice to other transparent conductive oxide (TCO) materials (sulphide-based phosphors) usually dedicated to optoelectronic devices. Various growth techniques have been used to prepare thin films of this material: electron beam evaporation [8], spray pyrolysis deposition [9], sol gel process [7] and radiofrequency (RF) magnetron sputtering [10].

In the present paper, we report the use of RF magnetron sputtering to prepare undoped $\beta-\mathrm{Ga}_{2} \mathrm{O}_{3}$ thin films. Structural characterizations, optical absorption as well as electrical conductivity properties are studied versus anneal treatments during and after the growth process. In addition, Nd-doped $\beta-\mathrm{Ga}_{2} \mathrm{O}_{3}$ thin films have been fabricated by co-sputtering and the first PL data show the emitting signatures of the rare earth element in the infrared spectral region.

2 Experimental details Gallium oxide $\mathrm{Ga}_{2} \mathrm{O}_{3}$ thin films were prepared by radio-frequency magnetron sputtering of a $\mathrm{Ga}_{2} \mathrm{O}_{3}(99.99 \%$ purity) target. Before any deposit, the chamber was evacuated down to $1.0 \times 10^{-7}$ Torr. The substrate temperature $\left(T_{\mathrm{s}}\right)$ during deposit was varied over the range $100-600{ }^{\circ} \mathrm{C}$. Then the layers were grown on single crystal silicon substrates under a pure argon pressure of $2.5 \times 10^{-2}$ Torr and a power density of $2.5 \mathrm{~W} / \mathrm{cm}^{2}$. The thickness of the deposited films ranged between $250 \mathrm{~nm}$ and $600 \mathrm{~nm}$. For each $T_{\mathrm{s}}$ value, (100)-oriented silicon sub- 
strates were placed in the chamber, side by side and at $7 \mathrm{~cm}$ just above the target. Post-annealing treatments were carried out at $T_{a}=900{ }^{\circ} \mathrm{C}$ and $1000{ }^{\circ} \mathrm{C}$ during 1 hour under a continuous flow of pure nitrogen. Nd-doping was achieved by placing a few pellets of Neodymium oxide on the target surface during the growth process corresponding to a surface ratio of about $5 \%$.

Various characterization techniques were used to analyse the evolution of the structural, optical and electrical properties of the films upon growth conditions $\left(T_{\mathrm{s}}\right)$ and post-anneal treatments $\left(T_{\mathrm{a}}\right)$. X-ray spectroscopy measurements were performed using a Philips XPERT diffractometer with $\mathrm{Cu} \mathrm{K}_{\alpha}(\lambda=1.5418 \AA)$ as X-ray source. Usual $\theta-2 \theta$ diagrams were recorded under a grazing incidence of $0.5^{\circ}$. Conventional and high resolution Transmission Electron Microscopy (TEM) observations of cross sectional views of the films were performed using a JEOL 2010F operated with a Field Emission Gun source at $200 \mathrm{kV}$, and equipped with a CCD camera. The ellipsometric spectroscopy (ES) data were collected while the incident light was scanned between $1.5 \mathrm{eV}$ and $5.0 \mathrm{eV}$ under an incidence angle of $66.2^{\circ}$. This was realized by means of a JobinYvon ellipsometer (UVISEL). The ES spectra consist in the measurement of $\psi$ and $\Delta$ ellipsometric angles defined from the fundamental equation of ellipsometry:

$$
r_{\mathrm{p}} / r_{\mathrm{s}}=\tan \psi \mathrm{e}^{i \Delta}
$$

where $r_{\mathrm{p}}$ and $r_{\mathrm{s}}$ are the complex reflection coefficients for parallel and perpendicular polarizations of the light, respectively. The sheet resistance is a measure of resistance of thin films that have a uniform thickness. It is applicable to two-dimensional systems where the thin film is considered to be a two dimensional entity and is equivalent to resistivity as used in three-dimensional systems. In a regular three-dimensional conductor, the resistance can be written as:

$$
R=\rho \frac{L}{e W},
$$

where $\rho$ is the resistivity, $L$ is the length, $W$ is the width and $e$ the sheet thickness. By grouping the resistivity with the thickness, the resistance can then be written as:

$$
R=R_{\mathrm{s}} \frac{L}{W} \quad \text { with } \quad R_{\mathrm{s}}=\frac{\rho}{e} .
$$

$R_{\mathrm{s}}$ is then the sheet resistance in ohms/square $(\Omega / \square)$. For a square, $L=W$ and therefore, $R=R_{\mathrm{s}}$. PL response was investigated at room temperature using an Ar laser line which induces a resonant excitation of $\mathrm{Nd}$ (operating at $514 \mathrm{~nm}$ ). The emission was recorded through a system of measurement composed by a chopper, a monochromator (Jobin-Yvon THR1000) followed by a cooled Ge detector and a lock-in amplifier. The PL measurement was carried out with a laser beam chopped at $45 \mathrm{~Hz}$ and an excitation power of $1.3 \mathrm{~W} / \mathrm{cm}^{2}$.

\section{Results and discussion}

3.1 Substrate temperature effect All the synthesised films were found to be amorphous whatever the chosen $T_{\mathrm{s}}$ value. The XRD patterns obtained for all the $T_{\mathrm{s}}$ values (not shown) give rise to similar spectra: two broad bands centred at about $32^{\circ}$ and $64^{\circ}$ are visible. These positions correspond approximately to the positions of the main diffraction peaks of $\beta-\mathrm{Ga}_{2} \mathrm{O}_{3}$. These broad bands could suggest the presence of a nanocrystalline structure of the films but TEM observations (not shown) showed that they were fully amorphous. The films were also subject to SE analyses by using a model dealing with an appropriate dispersion law such as Forouhi-Bloomer [11] and the Bruggeman effective medium approximation (EMA) [12]. Such analyses were already described elsewhere in the case of silicon nanoparticles embedded in a silica matrix [13].

The deduction of the optical parameters from the ellipsometric angles $\psi$ and $\Delta$ requires the reproduction of the experimental values in the energy range $1.5-5 \mathrm{eV}$. Although the EMA approach could appear more realistic for our material that might be considered as containing components of both crystalline and amorphous $\mathrm{Ga}_{2} \mathrm{O}_{3}$, it requires, however, the existence of reference spectra for these media. As example, we chose as a reference for silicon substrate, data contained in the software DELTAPSI2 from Jobin-Yvon. Concerning the $\mathrm{Ga}_{2} \mathrm{O}_{3}$ films, the dispersion law which models the dielectric function, called "new amorphous" has been used. It derives from the ForouhiBloomer law, commonly used in the case of both semiconducting and insulating materials. A nearly perfect agreement between the measured and calculated spectra was obtained and the thickness agrees well with that obtained by other techniques. However, the major data extracted from the modelling are the optical properties as will be discussed hereafter. Figure 1 depicts the static (in the infrared region) refractive index spectra $n$ and the deposition rate as

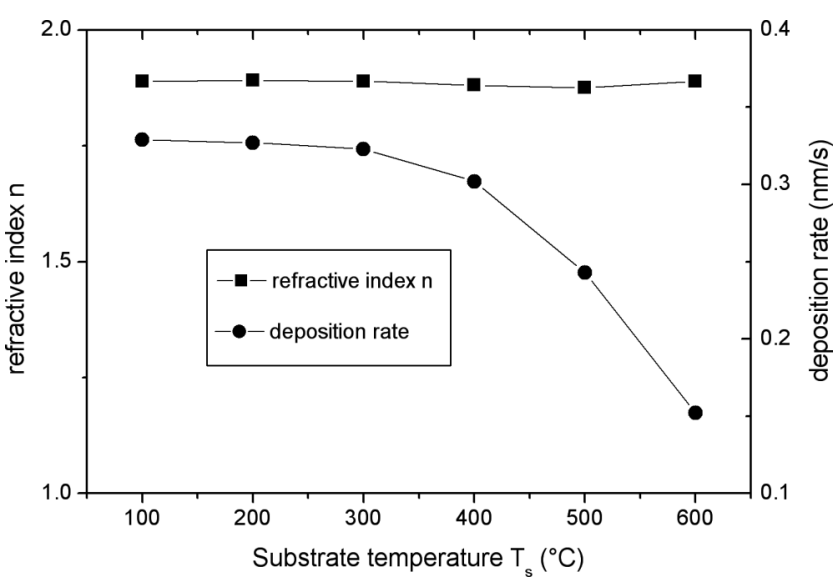

Figure 1 Static refractive index $(n)$ and deposition rate versus substrate temperature. $n$ is constant with a value (about 1.91) close to that of bulk $\mathrm{Ga}_{2} \mathrm{O}_{3}$ and the deposition rate is decreasing when $T_{\mathrm{s}}$ is raised. 


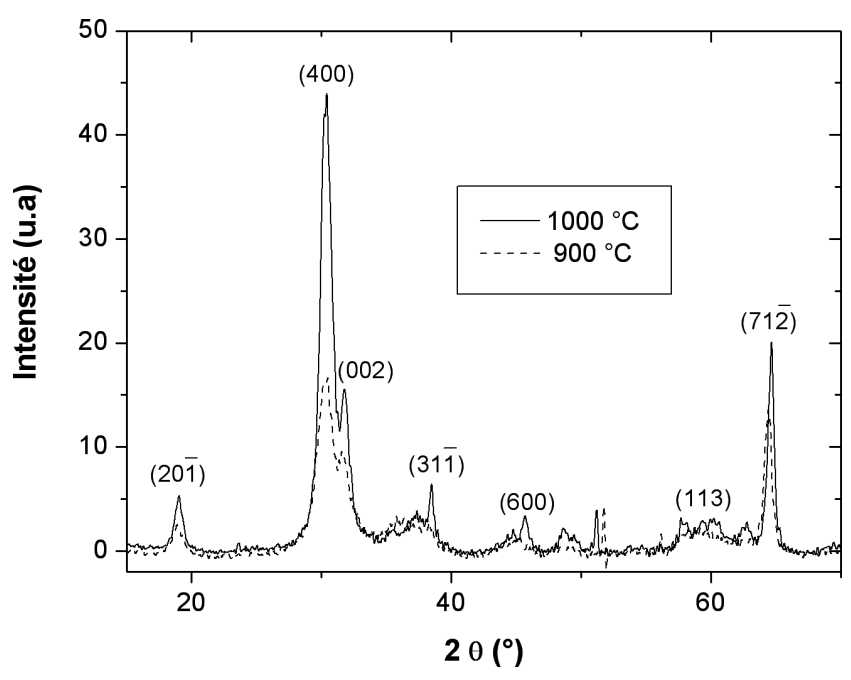

Figure 2 X-ray diffraction patterns of annealed gallium oxide films. Diffraction peaks positions are consistent with the monoclinic $\beta-\mathrm{Ga}_{2} \mathrm{O}_{3}$ structure. The high intensity of the (400) peak suggests a (400) texture of the film.

a function of the substrate temperature $T_{\mathrm{s}}$. Upon increasing $T_{\mathrm{s}}, n$ is almost constant (close to the value of bulk $\mathrm{Ga}_{2} \mathrm{O}_{3}$, $n=1.91$ ) whereas the deposition rate decreases in a monotonous way. This behaviour is usual and is explained by a larger reactivity of the species produced within the plasma when $T_{\mathrm{s}}$ increases [14].

3.2 Annealing temperature effect Anneal treatments were performed on films grown at $T_{\mathrm{s}}=100^{\circ} \mathrm{C}$. Figure 2 shows the XRD spectra of gallium oxide thin films annealed at $900{ }^{\circ} \mathrm{C}$ and $1000^{\circ} \mathrm{C}$ under a continuous flow of pure nitrogen. Diffraction peaks appear for both samples and their positions are consistent with the monoclinic structure of $\mathrm{Ga}_{2} \mathrm{O}_{3}\left(\beta-\mathrm{Ga}_{2} \mathrm{O}_{3}\right)$. However, higher intensities are observed for the sample annealed at $1000^{\circ} \mathrm{C}$ indicating a better formation of the $\beta-\mathrm{Ga}_{2} \mathrm{O}_{3}$ phase.

Compared to a diffraction spectrum of a powder $\beta-\mathrm{Ga}_{2} \mathrm{O}_{3}$ specimen, the intense (111) diffraction peak is missing and the highest intensity is observed for the peak related to the (400) planes suggesting thus a (400) preferred orientation of the films. There is no observable secondary phase in the XRD patterns. TEM observations led to an estimate of the grain size of a few tens of nanometres. Figure 3(a) is a typical HREM image of a film annealed at $1000{ }^{\circ} \mathrm{C}$. This region shows a grain oriented such that the Fourier transform in Fig. 3(b) is in accordance with the monoclinic structure of $\mathrm{Ga}_{2} \mathrm{O}_{3}$. The grain orientation is also consistent with the above mentioned (400) texture of the films.
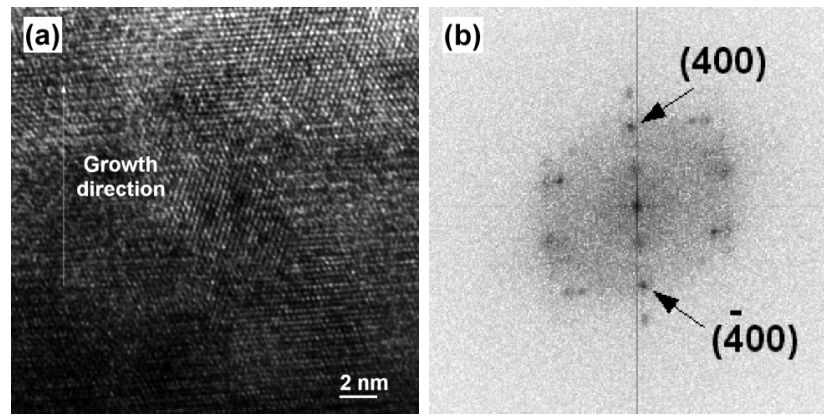

Figure 3 (a) HREM image of an annealed film at $1000{ }^{\circ} \mathrm{C}$, (b) Fourier transform of the image in Fig. 3(a). These data confirm the formation of $\beta-\mathrm{Ga}_{2} \mathrm{O}_{3}$ and the (400) texture of the films.

The $T_{\mathrm{a}}$ effect on the absorption coefficient $\alpha$ is shown in Fig. 4 which compares the $\alpha(E)$ spectra obtained from ellipsometry measurements for as-grown and annealed samples. These data reveal that all the samples are almost transparent below $3 \mathrm{eV}$ whereas a clear improvement appears when $T_{\mathrm{a}}$ increases.

However, close to $5 \mathrm{eV}$, the $T_{\mathrm{a}}$ effect is weak. This behaviour shows that the thin films become more transparent after post-annealing than for the as-grown sample. Annealing treatment allows certainly a crystallization of the films whose composition becomes close to that of the target. This seems to be confirmed by the electrical measurements. Indeed, Table 1 gives the values of the sheet resistances obtained for as-grown and annealed samples. One observes a decrease in resistance with $T_{\mathrm{a}}$ and thus indicates an improvement of the film conductivity. In Table 1 are also reported the absorption coefficient values at $E=2.3 \mathrm{eV}$ (corresponding to the medium of the visible range) and the transmission coefficients of the films calculated by $T=\mathrm{e}^{-\alpha x}$. It is then possible to calculate the figure of merit $\Phi_{\mathrm{TC}}$ of the transparent conductive sample by the following equation:

$$
\Phi_{\mathrm{TC}}=\frac{T^{10}}{R_{\mathrm{s}}} .
$$

The obtained values show that they are very satisfactory compared to those of other operating TCOs (about $1.0 \times 10^{-3}$ for $\mathrm{ZnO}$ [15], and $2.0 \times 10^{-3}$ for ZGO [16] and we can observe an increase of this factor with the annealing temperature.

Regarding the Nd-doped $\beta-\mathrm{Ga}_{2} \mathrm{O}_{3}$ films, optical properties are very close to those of non doped films. By EDX analysis, the Nd concentration does not exceed $1 \%$ corresponding to the detection limit of the spectrometer. Fig-

Table 1 Electrical and optical data of the gallium oxide films.

\begin{tabular}{lllllllll}
\hline sample & $L(\mathrm{~mm})$ & $e(\mathrm{~nm})$ & $W(\mathrm{~mm})$ & $R(\Omega)$ & $R_{\mathrm{s}}(\Omega / \square)$ & $\alpha\left(\mathrm{cm}^{-1}\right)$ & $T$ & $\Phi\left(\Omega^{-1}\right)$ \\
\hline as-grown & 10 & 544 & 5 & 3947 & 7895 & 431.0 & 0.98 & $1.0 \times 10^{-4}$ \\
$900^{\circ} \mathrm{C}$ & 10 & 590 & 5 & 3409 & 6818 & 7.4 & 1.00 & $1.5 \times 10^{-4}$ \\
$1000^{\circ} \mathrm{C}$ & 6 & 590 & 5 & 2113 & 2535 & 1370 & 0.93 & $1.9 \times 10^{-4}$ \\
\hline
\end{tabular}




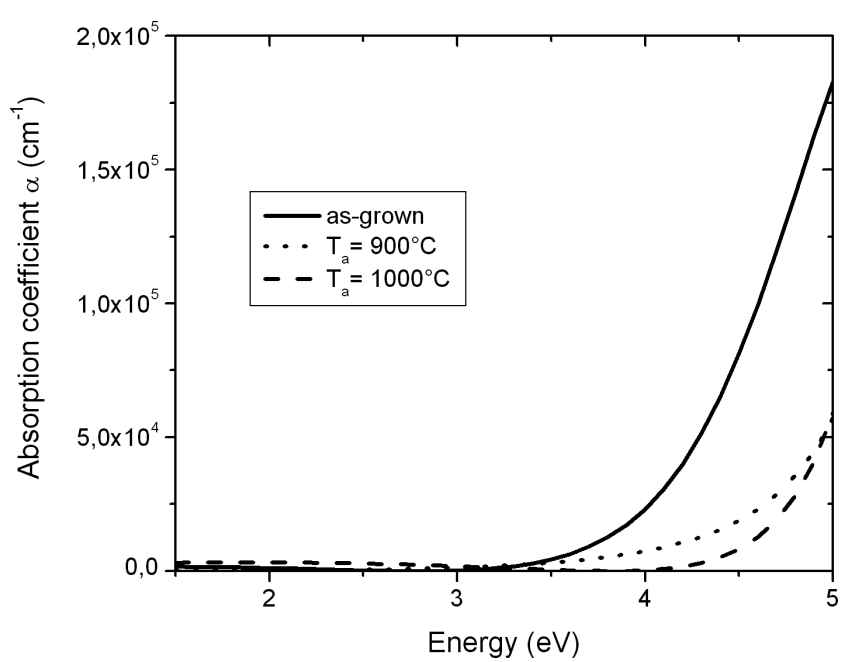

Figure 4 Dependence of the absorption coefficient on anneal temperature. The grown material is transparent in the visible range (from 1.6 up to $3.2 \mathrm{eV}$ ).

ure 5 exhibits the PL response of the Nd-doped $\beta-\mathrm{Ga}_{2} \mathrm{O}_{3}$ film annealed at $1000{ }^{\circ} \mathrm{C}$.

The rare-earth signature is clearly visible at $880 \mathrm{~nm}$ and $1070 \mathrm{~nm}$ corresponding respectively to $4 \mathrm{~F}$ transitions respectively $4 \mathrm{~F}_{3 / 2} \rightarrow 4 \mathrm{I}_{11 / 2}$ and $4 \mathrm{~F}_{3 / 2} \rightarrow 4 \mathrm{I}_{9 / 2}$ of $\mathrm{Nd}^{3+}$. This first result is encouraging and optimisation of the process is in progress. For instance, the effect of the number of pellets on the target as well as the anneal treatment has to be investigated and optimised. Very recently, a similar approach using a subdivided powder target into two or more parts led to multicomponent thin film phosphors for electroluminescent devices [17]. Our goal is to obtain similar results with our method.

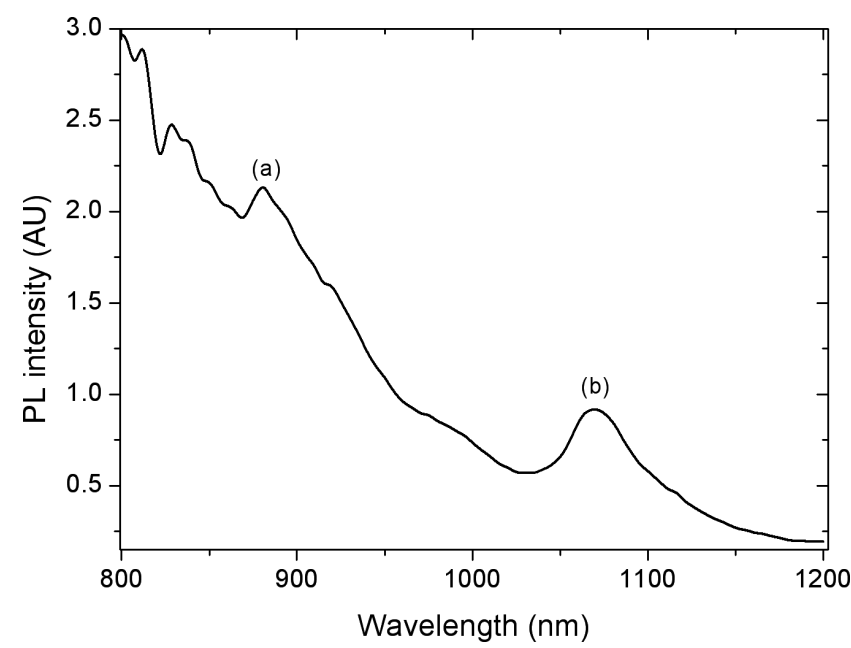

Figure $5 \mathrm{PL}$ spectrum for the $\mathrm{Nd}$-doped $\beta-\mathrm{Ga}_{2} \mathrm{O}_{3}$ film annealed at $1000{ }^{\circ} \mathrm{C}$. Nd signature is clearly identified by the presence of two peaks of luminescence at $880 \mathrm{~nm}$ (a) and $1070 \mathrm{~nm}$ (b).
4 Conclusion $\beta-\mathrm{Ga}_{2} \mathrm{O}_{3}$ polycrystalline thin films have been successfully grown by RF magnetron sputtering. The $\beta-\mathrm{Ga}_{2} \mathrm{O}_{3}$ phase appears upon an anneal treatment at about $900{ }^{\circ} \mathrm{C}$. The grain size is a few tens of nanometres and the films are (400) textured. A $T_{\mathrm{a}}$ increase favours a better figure of merit up to $1.9 \times 10^{-4}$ for $T_{\mathrm{a}}=1000^{\circ} \mathrm{C}$. A possible application of this material is transparent electrodes under extreme thermal conditions. Nd-doped films have been achieved with success since the typical emitting signals from $\mathrm{Nd}$ in the infrared region have been easily detected. This co-sputtering method of doping $\beta-\mathrm{Ga}_{2} \mathrm{O}_{3}$ films is then promising and will be optimised to fabricate light emitting diodes. These studies will be extended to other rare-earth elements.

\section{References}

[1] M. Passlack, E. F. Schubert, W. S. Hobson, M. Hong, N. Moriya, S. N. G. Chu, K. Konstadinis, J. P. Mannaerts, M. L. Schnoes, and G. J. Zidzik, J. Appl. Phys. 77, 686 (1995).

[2] T. Minami, Semicond. Sci. Technol. 20, 35 (2005).

[3] M. Fleisher and H. Meixner, Sens. Actuators B 4, 437 (1991).

[4] M. Passlack, N. E. J. Hunt, E. F. Schubert, G. J. Zidzik, M. Mong, J. P. Mannaerts, R. L. Opila, and R. J. Fischer, Appl. Phys. Lett. 64, 2715 (1994).

[5] E. Fortunato, V. Assunçao, A. Gonçalves, A. Marques, H. Aguas, L. Pereira, I. Ferreira, P. Vilarinho, and R. Martins, Thin Solid Films 451, 443 (2004).

[6] A. Shionoya and W. M. Yen, Phosphor Handbook (CRC Press, Boca Raton, FL, 1998).

[7] T. Miyata, T. Nakatani, and T. Minami, J. Lumin. 87-89, 1183 (2000).

[8] A. Callegari, P. D. Hoh, D. A. Duchanan, and D. Lacey, Appl. Phys. Lett. 54, 332 (1989).

[9] J. Hao and M. Cocivera, J. Phys. D, Appl. Phys. 35, 433 (2002).

[10] T. Minami, H. Yamada, Y. Kubota, T. Myata, and Y. Sakagami, Proc. of the Fourth International Display Workshop, 1997, p. 605

[11] A. R. Forouhi and I. Bloomer, Phys. Rev. B 34, 7018 (1989).

[12] D. A. G. Bruggeman, Ann. Phys. 24, 636 (1935).

[13] S. Charvet, R. Madelon, F. Gourbilleau, and R. Rizk, J. Appl. Phys. 85, 257 (1999).

[14] H. Colder, R. Rizk, M. Morales, P. Marie, and J. Vicens, J. Appl. Phys. 98, 24313 (2005).

[15] L. Raneiro, A. Gonçalves, A. Pimentel, I. Ferreira, S. Zhang, L. Pereira, H. Aguas, E. Fortunato, and R. Martins, Mater. Res. Soc. Symp. Proc. 862, A21.10.1 (2005).

[16] L. Raniero, I. Ferreira, A. Pimentel, A. Gonçalves, P. Canhola, E. Fortunato, and R. Martins, Thin Solid Films 511, 295 (2005)

[17] T. Minami, Y. Mochizuki, and T. Miyata, Thin Solid Films 494, 33 (2006). 